\title{
The LAMOST spectroscopic survey of stars in the Kepler field of view: Activity indicators and stellar parameters ${ }^{\star}$
}

\author{
Joanna Molenda-Żakowicz ${ }^{1, \star \star}$, Antonio Frasca ${ }^{2}$, Peter De Cat ${ }^{3}$, and Giovanni Catanzaro \\ ${ }^{1}$ Uniwersytet Wrocławski, Instytut Astronomiczny, ul. Kopernika 11, 51-622 Wrocław, Poland \\ ${ }^{2}$ INAF-Osservatorio Astrofisico di Catania, via S. Sofia, 78, 95123 Catania, Italy \\ ${ }^{3}$ Royal Observatory of Belgium, Ringlaan 3, 1180 Brussels, Belgium
}

\begin{abstract}
We summarize the results of the completed first round of the LAMOST-Kepler project, and describe the status of its on-going second round. As a result of the first round of this project, the atmospheric parameters $\left(T_{\text {eff }}, \log g\right.$, and $\left.[\mathrm{Fe} / \mathrm{H}]\right)$, the spectral classification (spectral type and luminosity class), and the radial velocities $(R V)$ have been measured for 51,385 stars. For 4031 stars, we were able to measure the projected rotational velocity, while the minimum detectable $v \sin i$ was $120 \mathrm{~km} \mathrm{~s}^{-1}$. For 8821 stars with more than one observation, we computed the $\chi$-square probability that the detected $R V$ variations have a random occurrence. Finally, we classified 442 stars as chromospherically active on the basis of the analysis of their $\mathrm{H} \alpha$ and Ca II-IRT fluxes. All our results have been obtained from the low-resolution $(R \sim 1800)$ spectroscopic observations acquired with the LAMOST instrument.
\end{abstract}

\section{LAMOST-Kepler project}

The LAMOST-Kepler project, described in detail by [1], is an activity carried out in the framework of the Kepler Asteroseismic Science Consortium (KASC). Started in 2011, this project aims at deriving the effective temperature $\left(T_{\text {eff }}\right)$, the surface gravity $(\log g)$, the metallicity $([\mathrm{Fe} / \mathrm{H}])$, the radial velocity $(R V)$, the projected equatorial rotational velocity $(v \sin i)$, and performing the MK spectral classification for stars in the nominal field of view of the Kepler space telescope (hereafter: the Kepler field, [2]). The LAMOST-Kepler project aims at completing and verification of the atmospheric parameters of stars provided in the Kepler Input Catalog (KIC, [3]).

Our activity is crucial for the in-depth studies of stars observed with Kepler because among the $13,123,717$ stars listed in the KIC, only 2,106,821 (i.e., $16 \%$ of the total sample) have the atmospheric parameters provided in that catalogue. Moreover, as shown by, e.g., [4, 5], the precision of the determinations of $T_{\text {eff }}, \log g$, and $[\mathrm{Fe} / \mathrm{H}]$ of solar-type stars in the KIC is too low for asteroseismic modelling. For stars of spectral type earlier than A, the situation is even worse since for them the discrepancies between $T_{\text {eff }}$ in the KIC and the values derived from spectroscopic observations amount to several thousand Kelvin (see [6-9]).

\footnotetext{
${ }^{\star}$ Based on observations collected with the Large Sky Area Multi-Object Fiber Spectroscopic Telescope (LAMOST) located at the Xinglong Observatory, China.

${ }^{\star}$ molenda@astro.uni.wroc.pl
} 


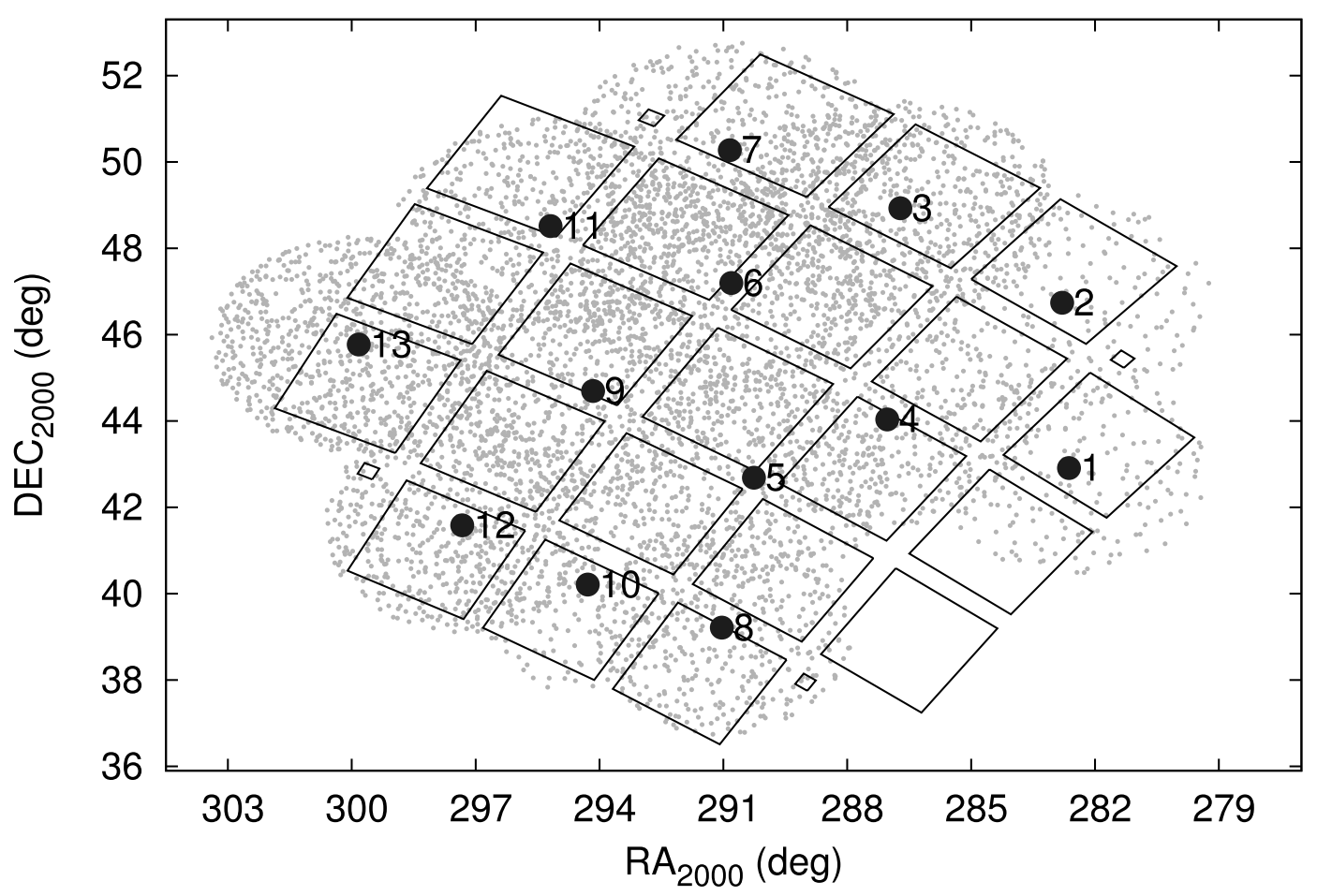

Figure 1. The nominal field of view of the Kepler space telescope covered with the LAMOST observations acquired in the second round of the LAMOST-Kepler project (in order to reduce the size of the figure, only every 15th target has been plotted). The lines indicate the location of the Kepler CCD modules. The gray dots indicate the location of the 86,378 observed stars. The large black dots indicate the centres of the 13 observed fields whose coordinates are provided in Table 1 .

In order to achieve the scientific goals of our project, we have been acquiring low-resolution $(R \sim 1800)$ spectroscopic observations using the Large Sky Area Multi-Object Fiber Spectroscopic Telescope (LAMOST) located at the Xinglong Observatory in China. We selected that instrument because it combines a large aperture (3.6-4.9 m) with a large, circular field of view of five degrees in diameter. This allows to cover the whole Kepler field with only 14 settings (see Fig. 2 in [1]). The focal surface of the LAMOST instrument is covered with 4000 optical fibers. Since some of them must be used for acquiring sky spectra and for calibration purposes, each exposure allows to acquire spectra of around 3000 stars (cf. Table 1). This gave us an opportunity to obtain spectroscopic observations for a significant number of stars from the Kepler field in a relatively short time.

\subsection{First round}

The first round of the LAMOST-Kepler project started in 2011 and ended in 2014. In that round we focused on stars which were included on the list of possible targets of the KASC. Based on those observations, in paper [10] we measured the atmospheric parameters and radial velocities of 51,385 stars. The average accuracy of these parameters is equal to $14 \mathrm{~km} \mathrm{~s}^{-1}$ in $R V, 3.5 \%$ in $T_{\text {eff }}, 0.3$ dex in $\log g$, and $0.2 \mathrm{dex}$ in $[\mathrm{Fe} / \mathrm{H}]$. In paper [10], we used the LAMOST data also to measure $v \sin i$ of 

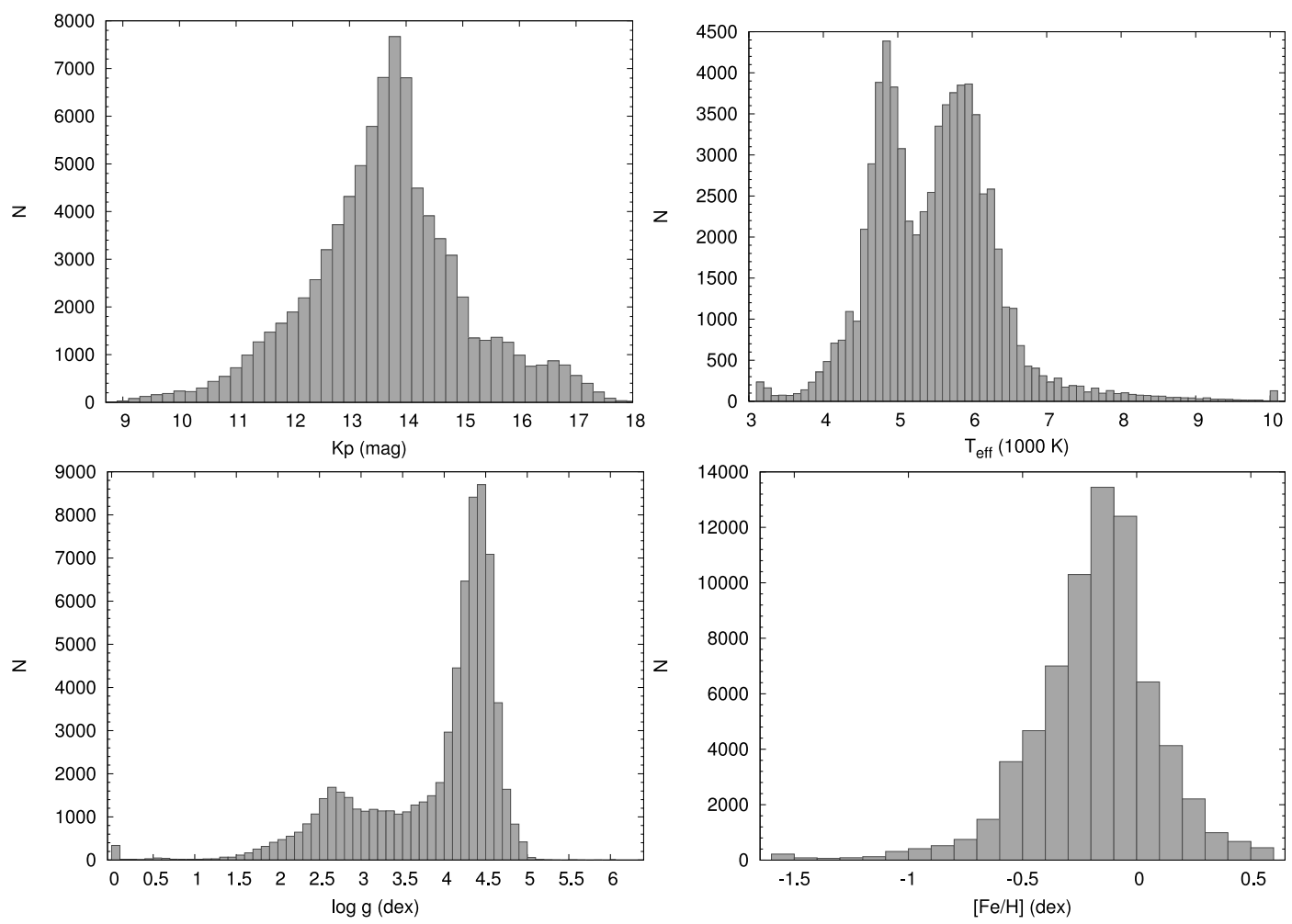

Figure 2. The global characteristics of the targets observed in the framework of the second round of the LAMOST-Kepler project. The histogram of the Kepler Kp magnitudes has been constructed for 86,284 stars. The histograms of $T_{\text {eff }}, \log g$, and $[\mathrm{Fe} / \mathrm{H}]$ have been constructed for 70,298 stars. The last bin of the histogram of $K \mathrm{p}$ includes 34 stars fainter than $18.1 \mathrm{mag}$. The last bin of the histogram of $T_{\text {eff }}$ includes 128 stars hotter than $10,000 \mathrm{~K}$. The first bin of the histogram of $\log g$ includes eight stars with $\log g<0$ dex. The first bin of the histogram of $[\mathrm{Fe} / \mathrm{H}]$ includes 134 stars with $[\mathrm{Fe} / \mathrm{H}]<-1.6$ dex. The $K \mathrm{p}, T_{\text {eff }}, \log g$, and $[\mathrm{Fe} / \mathrm{H}]$ values have been adopted from the Kepler Input Catalog.

stars that rotate with $v \sin i>120 \mathrm{~km} \mathrm{~s}^{-1}$. The latter value was found as the minimum detectable $v \sin i$ in our data from Monte Carlo simulations. For 547 stars which showed emission or filling-in the $\mathrm{H} \alpha$ line, we measured the equivalent width $(E W)$ of that line. The $E W$ of the Ca II $8498 \AA$ line has been measured for 348 stars, the $E W$ of the Ca II $8542 \AA$ line for 360 stars, and the $E W$ of the Ca II $8542 \AA$ line for 368 stars. The [NII] nebular lines have been detected in 55 stars. Based on the analysis of the $\mathrm{H} \alpha$ and Ca II-IRT fluxes of those 547 stars, we selected 442 stars that we classified as chromospherically active. We showed that one of them, KIC 8749284, is a likely accreting object.

\subsection{Second round}

The second round of the LAMOST-Kepler project started in 2015. In that round, we focused on stars which have already been observed with the Kepler telescope. In 2015, we acquired 97,641 spectra of 86,378 individual stars. 77,577 stars from that sample were observed only once, 7266 stars twice, 1195 stars three times, 254 stars four times, 66 stars five times, 14 stars six times, seven stars seven 
Table 1. The 13 settings of the LAMOST instrument used in the second round of the LAMOST-Kepler project. For each setting, the right ascension and declination of the centre of the respective field of view is provided along with the date(s) of observation, the number of exposures acquired for that field, and the total number of spectra that have been reduced from those data.

\begin{tabular}{cccccc}
\hline Field & R.A. (2000) & DEC (2000) & Date & $\begin{array}{c}\text { Number of } \\
\text { exposures }\end{array}$ & $\begin{array}{c}\text { Number of } \\
\text { spectra }\end{array}$ \\
\hline 1 & $18: 50: 31$ & $42: 54: 43$ & $2015-10-04$ & 1 & 3090 \\
2 & $18: 51: 11$ & $46: 44: 17$ & $2015-10-06$ & 1 & 2947 \\
3 & $19: 06: 51$ & $48: 55: 31$ & $2015-05-30$ & 3 & 7853 \\
$\prime \prime$ & $\prime \prime$ & $\prime \prime$ & $2015-09-15$ & 1 & 3084 \\
4 & $19: 08: 08$ & $44: 02: 10$ & $2015-09-25$ & 2 & 6153 \\
5 & $19: 21: 02$ & $42: 41: 13$ & $2015-10-11$ & 2 & 6223 \\
6 & $19: 23: 14$ & $47: 11: 44$ & $2015-09-14$ & 1 & 3140 \\
$\prime \prime$ & $\prime \prime$ & $\prime \prime$ & $2015-10-02$ & 2 & 6251 \\
7 & $19: 23: 23$ & $50: 16: 16$ & $2015-05-29$ & 2 & 5517 \\
$\prime \prime$ & $\prime \prime$ & $\prime \prime$ & $2015-09-13$ & 1 & 3169 \\
8 & $19: 24: 09$ & $39: 12: 42$ & $2015-10-12$ & 2 & 6303 \\
9 & $19: 36: 37$ & $44: 41: 41$ & $2015-09-21$ & 3 & 9163 \\
10 & $19: 37: 08$ & $40: 12: 49$ & $2015-10-18$ & 1 & 3120 \\
11 & $19: 40: 45$ & $48: 30: 45$ & $2015-09-16$ & 1 & 3135 \\
$\prime \prime$ & $\prime \prime$ & $\prime \prime$ & $2015-10-01$ & 2 & 6314 \\
12 & $19: 49: 18$ & $41: 34: 56$ & $2015-10-08$ & 3 & 9454 \\
13 & $19: 59: 20$ & $45: 46: 21$ & $2015-09-18$ & 2 & 6350 \\
$\prime \prime$ & $\prime \prime$ & $\prime \prime$ & $2015-10-03$ & 2 & 6375 \\
\hline \multicolumn{5}{c}{}
\end{tabular}

times, and one star eight times. The location of our targets in the Kepler field is shown in Figure 1. The numbers at the large black dots in Figure 1 refer to the centres of the 13 settings of the LAMOST instrument which were used for those observations. In Table 1, we provide the equatorial coordinates of those settings, the dates of observation, the number of exposures acquired in each setting, and the total number of spectra reduced from each setting.

Figure 1 shows that, in 2015, we covered almost the whole Kepler field with dense observations. The missing part and the sparsely sampled areas were scheduled for observing in 2016. However, bad weather conditions at the Xinglong Observatory in that year did not allow us to obtain the requested data. We expect to finish the second round of the LAMOST-Kepler project in 2017.

In Figure 2, we illustrate the global characteristics of the sample observed in 2015, adopting a similar approach to that used by us in paper [1] (see Fig. 6 therein). A visual comparison of the statistical properties of the stars observed in the first and the second round of the LAMOST-Kepler project shows that in the second round of the project, we observed stars falling in the same range of magnitude as in the first round, however, we included noticeably more stars fainter than 14 mag. The histograms of $T_{\text {eff }}, \log g$, and $[\mathrm{Fe} / \mathrm{H}]$ show that the properties of both samples, i.e. stars observed in the first and the second round, are similar. The quantitative analysis of the statistical properties of the sample observed in the second round will be carried out when the round is completed. By that time, we will also start the scientific analysis of the observations acquired in the second round of the LAMOST-Kepler project, which is planned to be a continuation of the work done in paper [10]. 
Acknowledgments: J.M.-Ż. acknowledges grant number NCN 2014/13/B/ST9/00902 and the Wrocław Centre for Networking and Supercomputing grant number 224.

\section{References}

[1] De Cat, P., Fu, J. N., Ren, A. B., et al., ApJS, 220, 19 (2015)

[2] Borucki, W., Koch, D., Boss, A., et al., in Second Eddington Workshop: Stellar structure and habitable planet finding, ed. F. Favata, S. Aigrain, \& A. Wilson, ESA SP-538, 117 (2004)

[3] Brown, T. M., Latham, D. W., Everett, M. E., \& Esquerdo, G. A., AJ, 142, 112 (2011)

[4] Creevey, O. L., Doğan, G.; Frasca, A., et al., A\&A, 537, 111 (2012)

[5] Stello, D., Chaplin, W. J., Bruntt, H., et al., ApJ, 700, 1589 (2009)

[6] Catanzaro, G., Frasca, A., Molenda-Żakowicz, J., \& Marilli, A\&A, 517, 3 (2010)

[7] Lehmann, H., Tkachenko, A., Semaan, T., et al., A\&A, 526, 124 (2011)

[8] McNamara, B. J., Jackiewicz, J., \& McKeever, J., AJ, 143, 101 (2012)

[9] Molenda-Żakowicz, J., Jerzykiewicz, M., Frasca, A., Catanzaro, G., Kopacki, G., \& Latham, D. W., preprint (arXiv: 1005. 0985) (2010)

[10] Frasca, A., Molenda-Żakowicz, J., De Cat, P., et al., A\&A, 594, 39 (2016) 